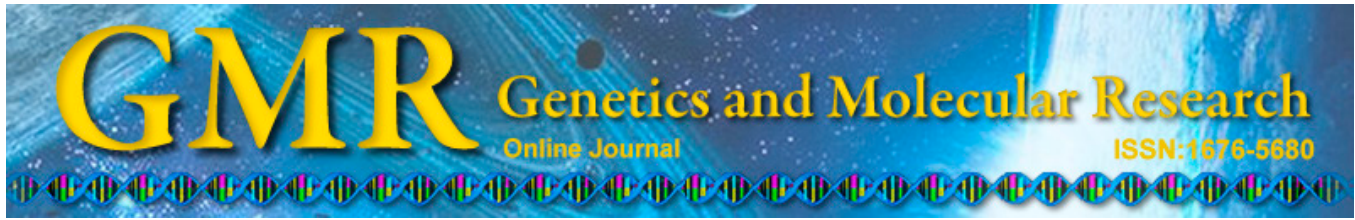

\title{
Associations between INSR and MTOR polymorphisms in type 2 diabetes mellitus and diabetic nephropathy in a Northeast Chinese Han population
}

\author{
A.N. Zhu' ${ }^{1 *}$ X.X. Yang ${ }^{1 *}$, M.Y. Sun ${ }^{2}$ Z.X. Zhang ${ }^{3}$ and M. Li ${ }^{1,3}$ \\ ${ }^{1}$ School of Biotechnology, Southern Medical University, Guangzhou, China \\ ${ }^{2}$ Guangzhou Center for Disease Control and Prevention, Guangzhou, China \\ ${ }^{3} \mathrm{Da}$ An Gene Co., Ltd. of Sun Yat-sen University, Guangzhou, China \\ *These authors contributed equally to this study. \\ Corresponding author: M. Li \\ E-mail: mingli2006_2006@126.com
}

Genet. Mol. Res. 14 (1): 1808-1818 (2015)

Received January 27, 2014

Accepted June 11, 2014

Published March 13, 2015

DOI http://dx.doi.org/10.4238/2015.March.13.9

ABSTRACT. We explored the associations of INSR and $m$ TOR, 2 key
genes in the insulin signaling pathway, and the susceptibility to type
2 diabetes mellitus and diabetic nephropathy. Three single-nucleotide
polymorphisms (SNPs) (rs 1799817 , rs 1051690 , and rs2059806)
in INSR and 3 SNPs (rs 7211818 , rs 7212142 , and rs9674559) in
$m T O R$ were genotyped using the Sequenom MassARRAY iPLEX
platform in 89 type 2 diabetes patients without diabetic nephropathy,
134 type 2 diabetes patients with diabetic nephropathy, and 120
healthy control subjects. Statistical analysis based on unconditional
logistic regression was carried out to determine the odds ratio (OR)
and $95 \%$ confidence interval (95\%CI) for each SNP. Combination
analyses between rs 2059806 and rs 7212142 were also performed
using the $\chi^{2}$ test and logistic regression. Among these 6 SNPs, 4
(rs 1799817 , rs 1051690 , rs 7211818 , and rs9674559) showed no 
association with type 2 diabetes mellitus or diabetic nephropathy. However, rs2059806 in INSR was associated with both type 2 diabetes mellitus $(\mathrm{P}=0.033)$ and type 2 diabetic nephropathy $(\mathrm{P}=$ $0.018)$. The rs 7212142 polymorphism in $m T O R$ was associated with type 2 diabetic nephropathy $(\mathrm{P}=0.010, \mathrm{OR}=0.501,95 \% \mathrm{CI}=0.288$ 0.871 ), but showed no relationship with type 2 diabetes mellitus. Combination analysis revealed that rs2059806 and rs7212142 had a combined effect on susceptibility to type 2 diabetes mellitus and diabetic nephropathy. Our results suggest that both INSR and mTOR play a role in the predisposition of the Han Chinese population to type 2 diabetic nephropathy, but the genetic predisposition may show some differences.

Key words: Diabetic nephropathy; INSR; MTOR; Polymorphisms; Type 2 diabetes mellitus

\section{INTRODUCTION}

Diabetes, particularly type 2 diabetes mellitus, continues to be a global health care problem. Kidney injury is a frequent and devastating complication of diabetes, particularly in chronic cases with poor nutritional control. Diabetic nephropathy is the major cause of chronic kidney disease and contributes significantly to increased morbidity and mortality among diabetic patients. It is also one of the most significant long-term complications and a leading cause of end-stage renal disease. The incidence and prevalence of end-stage renal failure in patients with type 2 diabetes has increased recently in both the Western world and Asia (Ritz, 1997). Investigations of candidate genes and genome-wide association studies have resulted in the identification of loci associated with genetic susceptibility to diabetic nephropathy (Tanaka and Babazono, 2005; Freedman et al., 2007) and type 2 diabetes mellitus (Wu et al., 2009; Liu et al., 2010; Ohshige et al., 2010). A genetic association study of type 2 diabetes mellitus and type 2 diabetic nephropathy will provide information regarding the pathogenesis of these 2 diseases, indicate those who are at risk, and provide new targets for therapy.

Genes involved in insulin signaling pathways are associated with susceptibility to type 2 diabetes mellitus (Laukkanen et al., 2004). Both INSR and MTOR play important roles in the insulin-signaling pathway (Avruch et al., 2005; Tremblay et al., 2005; Zick, 2005). The INSR gene encodes the insulin receptor, an important mediator between the extracellular and intracellular insulin signaling pathways, and is essential for the action of insulin. INSR is involved in both adipogenesis and beta-cell insulin secretion, but may also be involved in type 2 diabetes mellitus (Brunetti et al., 2011). Furthermore, INSR expression in the kidney changes during diabetes (Gatica et al., 2013), suggesting that it is a factor contributing to the development of type 2 diabetic nephropathy.

The metabolic actions of insulin are mediated by the insulin receptor/IRS/PI3-kinase signaling pathway (Ogawa and Kasuga, 2006). The protein mTOR belongs to the phosphatidylinositol 3-kinase (PI3K)-related kinase family and regulates inhibition of PI3K, which generates peptide growth factors central to the diverse responses elicited by insulin (Rui et al., 2001; Harrington et al., 2005). mTOR serves as an intracellular sensor for energy metabolism, 
nutrient availability, cellular and organism growth regulation, and metabolic adaptation to the environment. Furthermore, mTOR activation may play a pivotal role in the development of end-stage renal disease (Mori et al., 2009). Therefore, we suggest that mTOR signaling is a potential therapeutic target for type 2 diabetes mellitus and diabetic nephropathy (Sataranatarajan et al., 2007).

To explore the relationship between polymorphisms in INSR and $m T O R$ and the susceptibility to type 2 diabetes mellitus and type 2 diabetic nephropathy, 3 SNPs (rs1799817, rs1051690, and rs2059806) in INSR and 3 SNPs (rs7211818, rs7212142, and rs9674559) in mTOR were examined in a Han population from Northeast China using the MassARRAY iPLEX platform (Sequenom; San Diego, CA, USA).

\section{MATERIAL AND METHODS}

\section{Subjects}

A total of 134 diabetic nephropathy patients, 89 type 2 diabetes patients, and 120 healthy controls were enrolled between 2010 and September 2011 from Daqing Oil Field General Hospital in Northeast China. Type 2 diabetes patients were diagnosed according to the 2008 American Diabetes Association diagnostic criteria for diabetes and showed no evidence of renal dysfunction, with a disease course of more than 1 year. Type 2 diabetic nephropathy was confirmed in type 2 diabetes patients by renal biopsy in the absence of other causes of end-stage renal disease for more than 2 years. Control subjects were healthy, had no history of diabetes or other metabolic disorders, and were enrolled in the hospital during the same period. The study protocol was approved by the Clinical Research Ethics Committee of Nanfang Hospital, Southern Medical University. All subjects provided informed consent before participating in the study.

\section{DNA extraction}

Peripheral blood samples were drawn from the participants at Daqing Oil Field General Hospital in Northeast China. The samples were delivered frozen by express mail to the School of Biotechnology, Southern Medical University, Guangzhou, Guangdong Province, China, and stored at $-70^{\circ} \mathrm{C}$. Genomic DNA was extracted from $200 \mu \mathrm{L}$ peripheral blood using a Genomic DNA Purification kit (Tiangen Biotech; Beijing, China), according to the manufacturer instructions, and stored at $-70^{\circ} \mathrm{C}$ until use.

\section{Genotyping}

In accordance with the primary literature and the Single Nucleotide Polymorphism Database, rs1799817, rs1051690, and rs2059806 in INSR and rs7211818, rs7212142, and rs9674559 in MTOR were selected as candidate SNPs. All SNPs were genotyped using the SEQUENOM MassARRAY matrix-assisted laser desorption/ionization time-of-flight mass spectrometry platform (Sequenom; San Diego, CA, USA). Primers were designed using a semi-automated method (Assay Design 3.1, Sequenom). Detailed information and primer sequences of the SNPs are summarized in Table 1. 


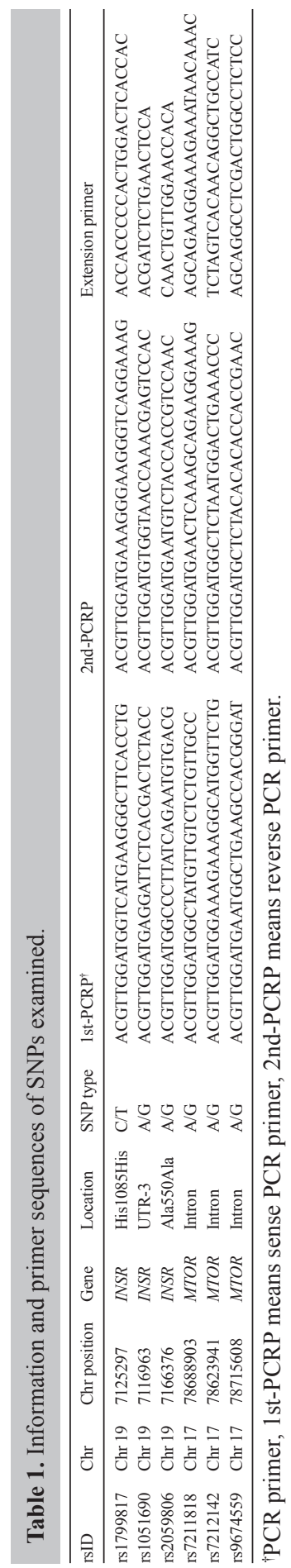




\section{Statistical analyses}

Hardy-Weinberg equilibrium for the 6 SNPs in the control group was assessed using the online tool SNPStats (http://bioinfo.iconcologia.net/SNPstats). Significant differences in genotype distributions between diabetic cases and controls were evaluated by $\chi^{2}$ analysis. Associations between the polymorphisms and disease risk were assessed using odds ratios (ORs) and $95 \%$ confidence intervals $(95 \% \mathrm{CIs})$ with binary logistic regression analyses, adjusted for age and gender. Statistical tests were implemented using the SPSS 13.0 software (SPSS, Inc.; Chicago, IL, USA). Linear regression analysis was used to assess the associations between genotype and clinical and biochemical characteristics. Combination analyses between rs2059806 and rs 7212142 were carried out using both the $\chi^{2}$ test and a logistic regression.

\section{RESULTS}

The characteristics of the 3 groups are shown in Table 2. A comparison among the 3 groups showed that age, body mass index, systolic blood pressure, diastolic blood pressure, and the fasting plasma glucose level were significantly different $(\mathrm{P}<0.05)$. There were significant differences in the urine protein, urine glucose, urea, and creatinine levels between the diabetic nephropathy and type 2 diabetes groups $(\mathrm{P}<0.001)$, while gender and $\mathrm{CO}_{2}$ binding were very similar $(\mathrm{P}=0.520, \mathrm{P}=0.598)$. Glycated hemoglobin was generally different $(\mathrm{P}=$ 0.054 ) between the 2 groups.

\begin{tabular}{|c|c|c|c|}
\hline & Control & T2DM* & $\mathrm{DN}^{* *}$ \\
\hline Male/Female & $61 / 59$ & $52 / 37$ & $75 / 59$ \\
\hline Age (years) & $52.7 \pm 6.44^{\dagger}$ & $56.80 \pm 11.87$ & $59.2 \pm 12.35$ \\
\hline BMI $\left(\mathrm{kg} / \mathrm{m}^{2}\right)$ & $23.38 \pm 2.74^{\dagger}$ & $25.02 \pm 3.40$ & $25.48 \pm 4.53$ \\
\hline $\mathrm{SBP}(\mathrm{mmHg})$ & $117.67 \pm 11.63^{\dagger}$ & $132.96 \pm 19.81$ & $146.69 \pm 29.14$ \\
\hline DBP (mmHg) & $78.58 \pm 7.81^{\dagger}$ & $80.40 \pm 11.27$ & $82.67 \pm 14.41$ \\
\hline Fasting plasma glucose (mM) & $4.77 \pm 0.48^{\dagger}$ & $9.58 \pm 3.78$ & $10.75 \pm 6.55$ \\
\hline \multicolumn{4}{|l|}{ Urine protein } \\
\hline- & $118(100.00 \%)$ & $75(88.23 \%)$ & $17(13.60 \%)$ \\
\hline+ & $0(0.00 \%)$ & $8(9.41 \%)$ & $33(26.40 \%)$ \\
\hline++ & $0(0.00 \%)$ & $2(2.35 \%)$ & $23(18.40 \%)$ \\
\hline+++ & $0(0.00 \%)$ & $0(0.00 \%)$ & $52(41.60 \%)$ \\
\hline Unknown & 2 & 4 & 9 \\
\hline \multicolumn{4}{|l|}{ Urine sugar } \\
\hline- & $118(100.00 \%)$ & $31(36.47 \%)$ & $45(35.43 \%)$ \\
\hline+ & $0(0.00 \%)$ & $7(8.23 \%)$ & $23(18.11 \%)$ \\
\hline++ & $0(0.00 \%)$ & $1(1.18 \%)$ & $13(10.24 \%)$ \\
\hline+++ & $0(0.00 \%)$ & $22(25.88 \%)$ & $35(27.56 \%)$ \\
\hline++++ & $0(0.00 \%)$ & $24(28.24 \%)$ & $11(8.66 \%)$ \\
\hline Unknown & 2 & 4 & 7 \\
\hline Glycated hemoglobin & $\mathrm{NA}^{\mathrm{a}}$ & $8.48 \pm 3.29$ & $7.70 \pm 2.08$ \\
\hline Urea $(\mathrm{mM})$ & NA & $6.53 \pm 2.15$ & $11.75 \pm 8.73$ \\
\hline $\mathrm{CO}_{2}$ binding $(\mathrm{mM})$ & NA & $25.29 \pm 3.89$ & $24.98 \pm 4.49$ \\
\hline Creatinine $(\mu \mathrm{M})$ & NA & $63.26 \pm 20.15$ & $198.63 \pm 236.34$ \\
\hline
\end{tabular}

*Type 2 diabetes patients without diabetic nephropathy. **Type 2 diabetes patients with diabetic nephropathy. ${ }^{\dagger}$ Data are reported as means \pm SD values. ${ }^{a} \mathrm{NA}$ means we did not collect the information.

The analysis of the 6 SNPs is shown in Table 3. The genotype distributions of the 4 SNPs rs1799817, rs 1051690, rs205980, and rs7212142 were consistent with Hardy-Weinberg equilibrium in the control group $(\mathrm{P}>0.05)$. Genotype frequencies for rs2059806 were sig- 
nificantly different among the 3 groups $(\mathrm{P}=0.043)$. The frequencies of genotypes $\mathrm{GG}$ and GA-AA of rs2059806 were 56.3 and $45.0 \%$ in controls, 71.1 and $28.9 \%$ in the type 2 diabetes group, and 54.9 and $45.1 \%$ in the diabetic nephropathy group, respectively. Compared with healthy controls, the GA-AA genotype of rs2059806 decreased the risk of type 2 diabetes mellitus, with an $\mathrm{OR}$ of $0.506(95 \% \mathrm{CI}=0.274-0.935, \mathrm{P}=0.030)$ after adjusting for age and gender. However, the GA-AA genotype showed an increased risk of diabetic nephropathy $(\mathrm{OR}=1.892,95 \% \mathrm{CI}=1.047-3.418, \mathrm{P}=0.035)$. The SNPs rs 1799817 and $\mathrm{rs} 1051690$ in INSR showed no differences among the 3 groups (Table 3 ).

For rs 7212142 , the distribution of GG-AA and GA differed among the 3 groups $(\mathrm{P}=$ 0.037). Of the control group, $57.1 \%$ of individuals carried genotype GG-AA and $42.9 \%$ had genotype GA, which was not significantly different from the type 2 diabetes group $(46.6 \%$ GG-AA and $53.4 \%$ GA) or diabetic nephropathy group (64.1\% GG-AA and $35.9 \%$ GA). However, compared with type 2 diabetes patients, the GA genotype of rs 7212142 decreased the risk of diabetic nephropathy $(\mathrm{OR}=0.501,95 \% \mathrm{CI}=0.288-0.871, \mathrm{P}=0.014$; Table 3$)$.

To evaluate the combined effect of rs 2059806 and rs 7212142 , the recombination of rs2059806 and rs7212142 was analyzed using an overall $\chi^{2}$ test using logistic regression. There were significant differences between genotypes (Table 4); the combined genotype GA/ AA-GG/AA showed greater protection against type 2 diabetes mellitus $(\mathrm{OR}=0.289,95 \% \mathrm{CI}$ $=0.116-0.721, \mathrm{P}=0.008)$ and an increased risk of diabetic nephropathy $(\mathrm{OR}=4.29,95 \% \mathrm{CI}=$ $1.17-10.63, \mathrm{P}=0.002$ ) compared with GA-AA of rs2059806. Any such combined effect may be caused by their biological interaction and the INSR and MTOR genes may function within a single pathway.

\section{DISCUSSION}

Diabetic nephropathy is a progressive renal disease and represents a serious late complication of diabetes (Lehmann and Spinas, 1995); 20-50\% of type 2 diabetic cases will develop into diabetic nephropathy. Multiple factors contribute to the pathogenesis of diabetic nephropathy resulting from metabolic abnormalities, hemodynamic alterations, and various growth and genetic factors (Parving et al., 1996). Insulin signaling occurs through the mitogen-activated protein kinase and PI3K pathways, via the insulin receptor in podocytes, and remodels podocyte cells (Welsh et al., 2010). Mutations in the insulin receptor may play roles in the pathogenesis of type 2 diabetes mellitus and diabetic nephropathy. The PI3K/AKT/MTOR pathway is important in diabetic nephropathy and MTOR signaling, and is activated in early diabetic nephropathy (Sakaguchi et al., 2006). mTOR is expressed primarily in renal podocytes, resulting in a number of deleterious effects such as hypertrophy of podocytes, which is critical for the development of diabetic nephropathy (Pagtalunan et al., 1997; Inoki et al., 2011).

In the present study, rs1799817 in exon 17 of INSR was not associated with disease. However, in South Indian individuals, this SNP is associated with type 2 diabetes mellitus (Bodhini et al., 2012). Within Mexican ethnic groups, rs2059806 showed no association with type 2 diabetes (Flores-Martinez et al., 2004). However, in our study, this SNP was not only associated with type 2 diabetes mellitus, but also with type 2 diabetic nephropathy. Furthermore, opposite associations were observed between this SNP and 2 other diseases. Compared with the AA genotype, the GA-AA genotype was associated with a 1.89-fold increased risk of diabetic nephropathy $(\mathrm{P}=0.018, \mathrm{OR}=1.89,95 \% \mathrm{CI}=1.05-3.42)$. The GA-AA genotype was a protective factor for type 2 diabetes mellitus $(\mathrm{P}=0.033, \mathrm{OR}=0.51,95 \% \mathrm{CI}=0.27-0.94)$. 


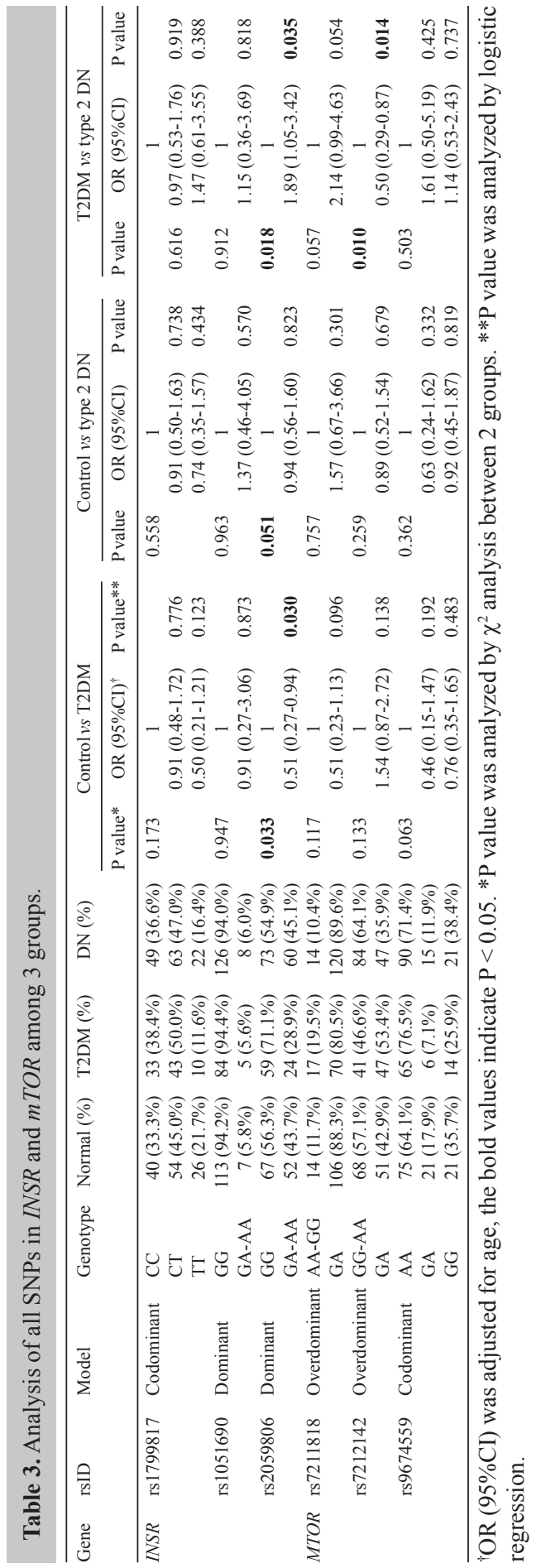




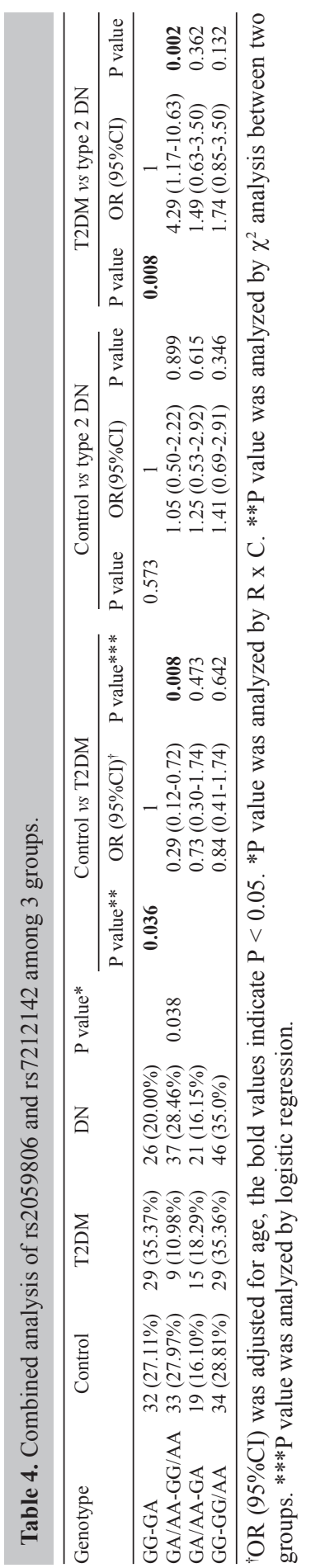


These results suggest that the GA-AA genotype plays opposing roles in the development of type 2 diabetes mellitus and diabetic nephropathy; in the development of diabetes, it is a protective factor, while this genotype increases the risk of diabetic nephropathy development. No other study has reported a susceptibility effect of rs 2059806 polymorphism on type 2 diabetes mellitus or diabetic nephropathy. These opposite results may be because of different pathogenic mechanisms of type 2 diabetes mellitus and diabetic nephropathy, possibly an innocent bystander of hyperinsulinemic states (Feliers et al., 2001) and hyperglycemia (Coward and Saleem, 2011). Multiple observations, such as elevated plasma and uric acid levels in type 2 diabetes, the reabsorption of which is correlated with the plasma insulin level, suggest that the kidney may not be insulin-resistant. As an index of insulin sensitivity, the insulin signaling pathway was activated in the renal cortex in type 2 diabetic mice (Feliers et al., 2001). Hyperinsulinism can lead to abnormal vasoreactivity, induce glomerular hyperfiltration, and contribute to albuminuria (Groop et al., 2005). Hyperglycemia can drive glucotoxicity of the kidney by increasing polyol and hexosamine levels, generating advanced glycation end products, activating signaling molecules, such as $\mathrm{PKC}$ and transforming growth factor- $\beta$ and plays a critical role in hyperglycemia-induced kidney damage (Schrijvers et al., 2004; Dronavalli et al., 2008). High extracellular glucose initiates podocyte apoptosis, a key feature of diabetic glomerular damage in vitro and in vivo by inducing intracellular reactive oxygen species (Susztak et al., 2006). Glycemic control is crucial for diabetic nephropathy prevention and treatment (Sakaguchi et al., 2006; Welsh et al., 2010). Therefore, strong cases can be made for hyperinsulinism and glycemia as the central pathogenic insults to the kidney. To highlight this, variation in insulin receptors is key to insulin action and INSR may be related to type 2 diabetic nephropathy.

mTOR plays an important role in many renal diseases, serving as an important modulator of renal repair and recovery (Lieberthal and Levine, 2009). In the present study, MTOR showed a relationship with only type 2 diabetic nephropathy. In an overdominant model, the GA genotype of rs 7212142 was significantly protective against diabetic nephropathy, with an OR of $0.501(95 \% \mathrm{CI}=0.288-0.871, \mathrm{P}=0.014)$, compared with the type 2 diabetes mellitus group. There was no significant difference between controls and the type 2 diabetes mellitus group or between controls and the diabetic nephropathy group. Activation of mTOR signaling caused renal hypertrophy at an early stage of diabetes (Sakaguchi et al., 2006), and high mTORC1 activation led to mislocalization of nephrin, which is a podocyte cell-surface protein important for the function of the renal filtration barrier (Lu et al., 2011). In diabetic rats, treatment with a low-dose sirolimus inhibitor of MTOR had a beneficial effect on diabetic kidneys, but did not modify symptoms induced by diabetes (Lloberas et al., 2006). Overall, MTOR plays an important role in the pathogenesis of diabetic nephropathy.

MTOR is important for podocytes and may be regulated by an insulin receptor by activating PI3K signaling. The combined effects of the 2 SNPs, rs 2059806 and rs 7212142 , were analyzed. The effect of the combined genotype GA/AA-GG/AA was different from GA-AA of rs2059806 and GG-AA of rs7212142, respectively, but was similar to the GA-AA genotype of rs2059806. The genotype GA/AA-GG/AA showed opposite effects on type 2 diabetes mellitus $(\mathrm{OR}=0.289,95 \% \mathrm{CI}=0.116-0.721)$ and diabetic nephropathy $(\mathrm{OR}=4.285,95 \% \mathrm{CI}=$ 1.172-10.631), which was not simply an additive or multiplicative effect; this may have arisen from the interaction between INSR and MTOR. This suggests that INSR, along with MTOR, contribute to type 2 diabetes mellitus and diabetic nephropathy. 
In conclusion, rs2059806 of INSR was associated with both type 2 diabetes mellitus and type 2 diabetic nephropathy, while rs 7212142 of $m T O R$ was associated with type 2 diabetic nephropathy but not type 2 diabetes mellitus. Combination analysis revealed that rs 2059806 and rs 7212142 have a cumulative effect on the susceptibility to type 2 diabetes mellitus and diabetic nephropathy. Whether the combined effects are caused by allele-allele interactions requires further investigation.

\section{Conflicts of interest}

The authors declare no conflict of interest.

\section{ACKNOWLEDGMENTS}

Research supported by the Research Fund for the Doctoral Program of Higher Education of China (Grant \#20104433120016) and the Youth Foundation of Southern Medical University (\#B1012022).

\section{REFERENCES}

Avruch J, Lin Y, Long X, Murthy S, et al. (2005). Recent advances in the regulation of the TOR pathway by insulin and nutrients. Curr. Opin. Clin. Nutr. Metab. Care 8: 67-72.

Bodhini D, Sandhiya M, Ghosh S, Majumder PP, et al. (2012). Association of His1085His INSR gene polymorphism with type 2 diabetes in South Indians. Diabetes Technol. Ther. 14: 696-700.

Brunetti A, Chiefari E and Foti D (2011). Perspectives on the contribution of genetics to the pathogenesis of type 2 diabetes mellitus. Recenti Prog. Med. 102: 468-475.

Coward RJ and Saleem MA (2011). Podocytes as a target of insulin. Curr. Diabetes Rev. 7: 22-27.

Dronavalli S, Duka I and Bakris GL (2008). The pathogenesis of diabetic nephropathy. Nat. Clin. Pract. Endocrinol. Metab. 4: 444-452.

Feliers D, Duraisamy S, Faulkner JL, Duch J, et al. (2001). Activation of renal signaling pathways in $\mathrm{db} / \mathrm{db}$ mice with type 2 diabetes. Kidney Int. 60: 495-504.

Flores-Martinez SE, Islas-Andrade S, Machorro-Lazo MV, Revilla MC, et al. (2004). DNA polymorphism analysis of candidate genes for type 2 diabetes mellitus in a Mexican ethnic group. Ann. Genet. 47: 339-348.

Freedman BI, Bostrom M, Daeihagh P and Bowden DW (2007). Genetic factors in diabetic nephropathy. Clin. J. Am. Soc. Nephrol. 2: 1306-1316.

Gatica R, Bertinat R, Silva P, Carpio D, et al. (2013). Altered expression and localization of insulin receptor in proximal tubule cells from human and rat diabetic kidney. J. Cell. Biochem. 114: 639-649.

Groop PH, Forsblom C and Thomas MC (2005). Mechanisms of disease: pathway-selective insulin resistance and microvascular complications of diabetes. Nat. Clin. Pract. Endocrinol. Metab. 1: 100-110.

Harrington LS, Findlay GM and Lamb RF (2005). Restraining PI3K: mTOR signalling goes back to the membrane. Trends Biochem. Sci. 30: 35-42.

Inoki K, Mori H, Wang J, Suzuki T, et al. (2011). mTORC1 activation in podocytes is a critical step in the development of diabetic nephropathy in mice. J. Clin. Invest. 121: 2181-2196.

Laukkanen O, Pihlajamäki J, Lindström J, Eriksson J, et al. (2004). Common polymorphisms in the genes regulating the early insulin signalling pathway: effects on weight change and the conversion from impaired glucose tolerance to Type 2 diabetes. The Finnish Diabetes Prevention Study. Diabetologia 47:871-877.

Lehmann R and Spinas GA (1995). Diabetic nephropathy: significance of microalbuminuria and proteinuria in Type I and Type II diabetes mellitus. Praxis 84: 1265-1271.

Lieberthal W and Levine JS (2009). The role of the mammalian target of rapamycin (mTOR) in renal disease. J. Am. Soc. Nephrol. 20: 2493-5202.

Liu LM, Zheng TS, Wang F, Wang NS, et al. (2010). Pro12Ala polymorphism in the PPARG gene contributes to the development of diabetic nephropathy in Chinese type 2 diabetic patients. Diabetes Care 33: 144-149. 
Lloberas N, Cruzado JM, Franquesa M, Herrero-Fresneda I, et al. (2006). Mammalian target of rapamycin pathway blockade slows progression of diabetic kidney disease in rats. J. Am. Soc. Nephrol. 17: 1395-1404.

Lu MK, Gong XG and Guan KL (2011). mTOR in podocyte function: is rapamycin good for diabetic nephropathy? Cell Cycle 10: 3415-3416.

Mori H, Inoki K, Masutani K, Wakabayashi Y, et al. (2009). The mTOR pathway is highly activated in diabetic nephropathy and rapamycin has a strong therapeutic potential. Biochem. Biophys. Res. Commun. 384: 471-475.

Ogawa W and Kasuga M (2006). Insulin signaling and pathophysiology of type 2 diabetes mellitus. Nihon Rinsho. 64: 1381-1389.

Ohshige T, Tanaka Y, Araki S, Babazono T, et al. (2010). A single nucleotide polymorphism in KCNQ1 is associated with susceptibility to diabetic nephropathy in japanese subjects with type 2 diabetes. Diabetes Care 33:842-846.

Pagtalunan ME, Miller PL, Jumping-Eagle S, Nelson RG, et al. (1997). Podocyte loss and progressive glomerular injury in type II diabetes. J. Clin. Invest. 99: 342-348.

Parving HH, Tarnow L and Rossing P (1996). Genetics of diabetic nephropathy. J. Am. Soc. Nephrol. 7: 2509-2517.

Ritz E (1997). Nephropathy in type II diabetes. Exp. Clin. Endocrinol. Diabetes 105 (Suppl 2): 80-82.

Rui L, Fisher TL, Thomas J and White MF (2001). Regulation of insulin/insulin-like growth factor-1 signaling by proteasome-mediated degradation of insulin receptor substrate-2. J. Biol. Chem. 276: 40362-40367.

Sakaguchi M, Isono M, Isshiki K, Sugimoto T, et al. (2006). Inhibition of mTOR signaling with rapamycin attenuates renal hypertrophy in the early diabetic mice. Biochem. Biophys. Res. Commun. 340: 296-301.

Sataranatarajan K, Mariappan MM, Lee MJ, Feliers D, et al. (2007). Regulation of elongation phase of mRNA translation in diabetic nephropathy:amelioration by rapamycin. Am. J. Pathol. 171: 1733-1742.

Schrijvers BF, De Vriese AS and Flyvbjerg A (2004). From hyperglycemia to diabetic kidney disease: the role of metabolic, hemodynamic, intracellular factors and growth factors/cytokines. Endocr. Rev. 25: 971-1010.

Susztak K, Raff AC, Schiffer M and Böttinger EP (2006). Glucose-induced reactive oxygen species cause apoptosis of podocytes and podocyte depletion at the onset of diabetic nephropathy. Diabetes 55: 225-233.

Tanaka N and Babazono T (2005). Assessing genetic susceptibility to diabetic nephropathy. Nephrology 10 (Suppl): S17-S21.

Tremblay F, Jacques H and Marette A (2005). Modulation of insulin action by dietary proteins and amino acids:role of the mammalian target of rapamycin nutrient sensing pathway. Curr. Opin. Clin. Nutr. Metab. Care 8: 457-462.

Welsh GI, Hale LJ, Eremina V, Jeansson M, et al. (2010). Insulin signaling to the glomerular podocyte is critical for normal kidney function. Cell. Metab. 12: 329-340.

Wu LS, Hsieh CH, Pei D, Hung YJ, et al. (2009). Association and interaction analyses of genetic variants in ADIPOQ, ENPP1, GHSR, PPARgamma and TCF7L2 genes for diabetic nephropathy in a Taiwanese population with type 2 diabetes. Nephrol. Dial. Transplant. 24: 3360-3366.

Zick Y (2005). Ser/Thr phosphorylation of IRS proteins: a molecular basis for insulin resistance. Sci. STKE 2005: pe4. 